\title{
Aggressive, Metastatic Squamous Cell Carcinoma After a 46-Year-Old History of Chronic Osteomyelitis and Local Infectious Complications: A Case Report
}

\begin{abstract}
Marjolin's ulcer is a squamous cell carcinoma (SCC) arising from any site of established chronic inflammation, especially with presence of scar tissue.

An emblematic case report of locally disseminated SCC arising from a chronic osteomyelitis of the left leg complicated by recurring soft tissue infections lasting since 46 years is presented and discussed according to the available international literature evidence. Concurrent diseases, supporting factors, clinical and histopathological presentation, differential diagnosis, and time and mode of management of this potentially functional- and life-threatening pathological condition are reviewed and discussed to offer a theme to daily clinical care.
\end{abstract}

Keywords: Carcinoma, Squamous Cell; Osteomyelitis; Infection; Local; Surgery; Marjolin's disease

Carcinoma squamocellulare metastatico aggressivo insorto dopo una storia di 46 anni di osteomielite cronica e di complicanze infettive locali: un caso clinico

CMI 2018; 12(1): 31-36

https://doi.org/10.7175/cmi.v12i1.1352

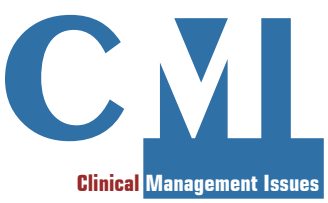

Case Report
Department of Infectious

Diseases,

"Alma Mater Studiorum"

University of Bologna,

S. Orsola-Malpighi

Hospital,

Bologna, Italy

2 Department of

Orthopedics,

S. Orsola-Malpighi

Hospital,

Bologna, Italy

\section{INTRODUCTION}

Skin tumors are the most frequent cancers in the world [1]. Even though ultraviolet radiation coming from the sun is the main responsible, scars are recognized as another important cause of tumorigenesis.

Among skin cancers arising in scars, squamous cell carcinomas-SCCs are the most frequent (71\%), followed by basal cell carcinomas-BCCs $(12 \%$, more common in male patients), and cutaneous melanomas $(6 \%$, more common in female patients) $[1,2]$. They mainly arise in burns experienced during childhood.

Marjolin's disease refers to an infrequent, aggressive ulcerating SCC presenting in a body surface area which has been previously interested for a long time by traumatized, chronically inflamed, infected, or scarred skin [3-5]. The term "Marjolin's ulcer" was named after the French surgeon Jean-Nico- las Marjolin (born 1780, died 1850), who reported for the first time this condition in the year 1828, when assessing non-healing ulcers developing in burn scars [6,7]. The term was later enforced by J.C. De Costa, as reported by the dermatologist C. Steffen in the year 1984 [6].

Although the mentioned Author initially did not specifically describe cancer or SCC [6], the term "Marjolin's ulcer" is now com-

\section{Why do we Describe This Case}

Patients affected by chronic osteomyelitis are treated and monitored for a long time. However, often physicians don't consider the underlying risk of local and disseminated carcinogenesis. Conversely, a prompt detection could be greatly beneficial for patients' survival
Corresponding author Dr. Roberto Manfredi, MD Infectious Diseases, University of Bologna, S. Orsola-Malpighi Hospital Via Massarenti 11 I 40138 Bologna, Italy

E-mail: roberto.manfredi@unibo.it

Received: 16 March 2018

Accepted: 4 May 2018

Published: 17 May 2018 


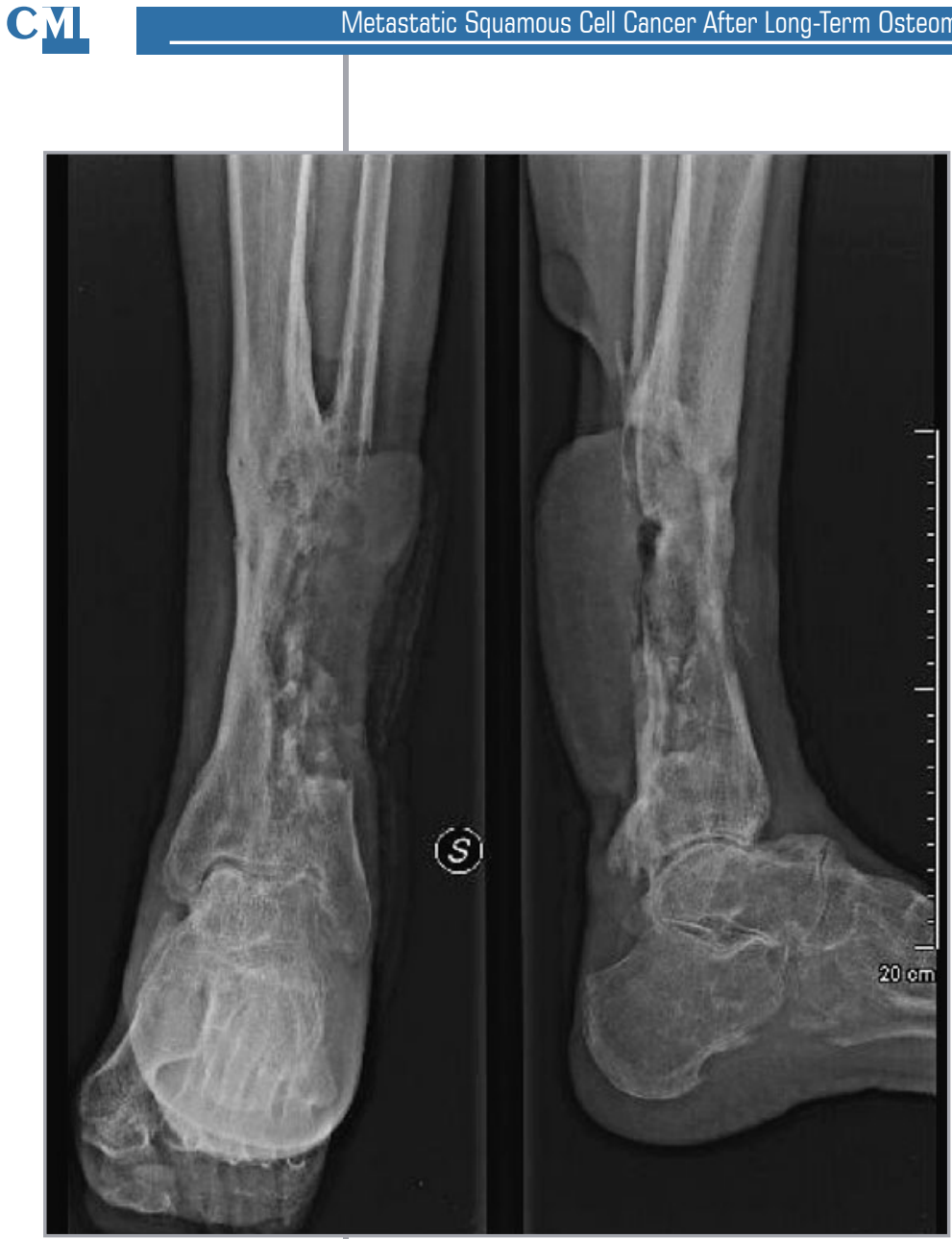

Figure 1. Chronic osteomyelitis of the distal portion of the left leg of our patient. The $X$-ray image shows a structural bone alteration of the distal tibia, with areas of bone rarefaction close to areas of bone sclerosis due to the sequelae of the inveterate osteomyelitic process. Inside the osteomyelitic areas, a remarkable osteolysis is appreciable, which leads to the disappearance of the distal portion of fibula, and that of the cortical, posterior-lateral tibial margins, attributable to the invasion by the overwhelming SCC of the skin. monly used to describe a cutaneous (squamous cell) carcinoma arising in any site of established chronic inflammation, especially with presence of scar tissue [3-5,8]. Even though a relevant number of Marjolin's ulcers still develop from burn scars, other sites include stasis ulcers, decubitus ulcers, inguinal granuloma, syphilitic lesions, and smallpox vaccination scars. They also may occur in sinus tracts from hidradenitis suppurativa, acne conglobata, and especially osteomyelitis (and its chronic draining sinuses and fistulae caused by infectious processes and superinfections, as well as long-lasting ulcerating scar tissue of non-united fractures) [3,9-14].

Here we report a case of severe SCC complicated by metastatic localizations, which led to leg amputation in a patient with an extremely long history of chronic osteomyelitis who underwent numerous medical and surgical interventions during a 46-year period.

\section{CASE DESCRIPTION}

A 62-year-old male patient had a 46-yearlong history of chronic left tibial and peroneal osteomyelitis, after a traumatic, severe, and exposed fracture occurred at the age of 16 . Since the first episode, the event became complicated by an osteomyelitis, and a relapsing, complicated, and sometimes gangrenous infection of soft tissues close to the previous bone fracture site.

Over 20 orthopedic/surgical interventions became necessary to stabilize the bone continuity, and to remove relapsing local infectious foci. In fact, despite endless cycles of antimicrobial chemotherapy, treatment with hyperbaric oxygen therapy, and repeated surgical stabilization and curettage interventions, relapsing episodes of fistulation occurred in the recent three years, with isolation of different bacterial microorganisms. Before hospitalization in our Department of Infectious Diseases, two Gram-negative multiresistant pathogens including Pseudomonas aeruginosa and Citrobacter freundii were cultured from a draining fistula, and the infection was treated with combined piperacillin-tazobactam (18 g/day in 24-hour i.v. continued infusion), and levofloxacin (500 $\mathrm{mg}$, twice daily orally).

On the ground of the slowly progressive improvement of both local and systemic signs of infection (with reduced secretions, almost normalized serum $\mathrm{C}$-reactive protein levels and other inflammation indexes), orthopedics and plastic surgeons finally evaluated the possibility of a combined approach, aimed at eliminating residual necrotic areas and bone sequestrations, checking and eventually enhancing tissue vascularization, and trying a reconstructive surgical approach of skin and skin structures. In this view, an arteriography was also performed, which showed an obstruction of the proximal tract of the left interbone artery, and two moderate stenotic tracts of the median third of posterior tibial artery.

At the time of hospitalization in our Department of Infectious Diseases, the patient suffered from a severe local pain, and presented enlarged ulcerations with a necrotic basis and abundant malodorous secretions at his left leg, in absence of fever and systemic signs and symptoms. Antibiotic treatment included i.v. piperacillin-tazobactam at $18 \mathrm{~g} /$ day, plus levofloxacin $500 \mathrm{mg}$, two times a day. The intense pain was poorly controlled by opioids, plus paracetamol, and gabapentin for paresthesia. A computerized tomography (CT) of the left leg disclosed a remarkable rarefaction of bone tissue, especially of the peroneal one, which contraindicated an eventual bone reconstruction. The underlying neoplastic complication was al- 
ready suspected by a conventional X-ray study of the limb (Figure 1).

During the same week, a sequestrectomy was performed with removal of necrotic remnants of leg bones and close tissues, together with a further revision of soft tissue infection foci, completed with a skin biopsy.

Unfortunately, the skin biopsy of the chronic cutaneous ulcer and the subsequent histopathological studies confirmed an already extensive and infiltrating SCC. An immediate staging of this malignancy was performed by a thoracic, abdominal, and pelvis $\mathrm{CT}$, and a positron-emission tomography (PET), which identified multiple enlarged regional lymph nodes. Our patient immediately underwent local surgery for skin cancer and local lymph node removal, but histopathology studies disclosed that all left inguinal lymph nodes, and left internal and external iliac artery lymph nodes tested positive for multiple metastatic localizations of the SCC. As a consequence, an amputation of the left leg at his upper third was promptly performed, and the treatment of cancer has been completed with multiple local radiotherapy cycles. A chemotherapy approach will be based upon an instrumental re-staging of the advanced neoplastic disease. At the time of writing, our patient is still undergoing his therapeutic pathway.

\section{DISCUSSION}

A local transformation of persistently inflamed cutaneous tissue close to scars and chronic inflammatory and infectious processes (including osteomyelitis and its complications) may be retrieved with stable or increasing frequency in the context of chronic wounds including burn injuries, skin ulcers descending from burns [4], venous stasis, post-radiotherapy scars, and especially ulcers from chronic osteomyelitis and its long-lasting sequelae [3,11], as also confirmed by extensive Italian experiences conducted in the reference tertiary center of Bologna, Italy (Rizzoli Orthopedic University Hospital) [12,13].

Anyhow, the intrinsic pathogenetic pathways for this malignant transformation are still poorly known. One suggestion is that previously traumatized skin may be more susceptible to mutation, and that fibrotic and avascular scar tissue may interfere with im- mune surveillance, resulting in the inability of peripheral lymphocytes and monocytemacrophage cells to recognize, reach, and destroy neoplastic cells [15].

In a nosographic perspective, R.J. Esther [3] underlined some major differences between a "true" Marjolin's ulcer, and a malignant skin transformation arising in a sinus of chronic osteomyelitis. In particular, according to this Author:

- in the event of Marjolin's ulcer, the size of lesion is large, the lymph nodes are involved, and the prognosis is poor;

- in the event of osteomyelitis sinuses, the size of lesion is small, the lymph nodes are not involved, and the outcome is more favorable [3].

However, both clinical and pathological presentation and outcome of these two proposed forms of malignant degeneration of chronic cutaneous ulcerative/inflammatory lesions are largely overlapping in the daily clinical practice, as demonstrated by our case report, where prominent osteomyelitis-associated complications, bone sequestrations, and draining fistulations were present, together with ulcerating skin lesions and multiple superinfections: the prognosis was poor, due to an extensive lymph node spread of SCC.

Anyway, a SCC developing on chronic skin lesions seems to have a greater incidence of metastasis (estimated to occur in $9 \%$ to $36 \%$ of cases), as compared to the same form of carcinoma arising in previously normal skin (with secondary lymph node localizations complicating $1 \%$ to $10 \%$ of episodes). Squamous skin cancer which develops on top of draining sinuses and ulcers seems to be more aggressive than the sensu stricto "Marjolin's ulcer" of skin scars occurring on previous burns, in absence of concurrent local inflammatory diseases, like a chronic osteomyelitis $[16,17]$.

From a clinical point of view, the disease presents as a slowly growing, painless ulcerated lesion, since the ulcer is usually not associated with nerve tissue involvement, so that it is usually indolent, thus leading to a frequently missed or delayed diagnosis. The lesions exhibit exuberant granulation tissue that spills over their well-defined margins onto the surrounding tissue. In around 40\% of cases these lesions involve the lower extremities, and since the malignant change of these long-lasting wounds is usually painless, and happens a very long time after initial trauma or local disease (up to 20-32 years 
later, with one case reported 70 years after the supporting disease had been identified) [18], its evolution is particularly insidious and may become life-threatening (in up to $80 \%$ of cases), if it is not recognized and treated promptly.

In particular, Marjolin's ulcers and related diseases have a particularly aggressive course (especially when compared with their extremely slow transformation process), enclosing greater rates of recurrence and overall metastatic lesions, as opposed to other forms of SCC. As a consequence, the estimated five-year survival rate of involved patients is around $30 \%$ only.

Actually, the SCC is per se the second leading cause of skin cancer death after melanoma, and it represents the second most common type of cutaneous cancer after basal cell carcinoma. Moreover, the time of development of malignancy is inversely proportionally to patient's age, so that also this last feature, together with the characteristic indolent-chronic appearance, often leads to a delayed recognition, and a consequently worse outcome.

The clinical-pathological differential diagnosis of these cases of "secondary" SCC and Marjolin's ulcer is extremely broad, and should include any non-healing ulcer such as venous stasis ulcers, diabetic ulcers, arterialvascular ulcers, pyoderma gangrenosum, or ulcerative cutaneous lesions secondary to other disease (i.e. burns, osteomyelitis, and multiple chronic dermatological disorders) [3,9-11,14].

A wedge biopsy of the lesion is the more affordable method of diagnosis. Tissue specimens obtained should be taken from both the center and margins of suspected lesions, as the central ulcerative areas may often show only necrotic material. Histologically, the tumor is represented by a welldifferentiated SCC in around 95\% of cases, although basal cell carcinoma, melanoma, and sarcomas may be also present occasionally. The malignancy is aggressive in nature, since it usually spreads locally to close areas, and is associated with a poor functional or life prognosis in many cases $[7,12]$. The local lymphatic vessel disruption has been thought to preserve from a lymphatic spread of cancer cells [12,19], but this event does not happen in long-term, advanced disease presentations, like ours.

Lifeso and Bull introduced a three-grade histopathological classification of SCC of the extremities: grade I (well differentiated form), grade II (moderately differentiated), and grade III (poorly differentiated form), and they found that this staging played a very relevant prognostic role [9].

There is limited evidence on what percentage of treatment-resistant, non-healing ulcers reveals a malignancy. Thus, experts recommend biopsy (i.e. wedge-shaped biopsy of the borders or multiple punch biopsies), of any non-healing ulcer resistant to standard interventions, to rule out malignant transformation.

Once diagnosed, wide excision followed by skin grafting is recommended. The radical treatment is based on a timely surgical excision, with a wide removal of the lesion, deserving margins of at least $1 \mathrm{~cm}$ all around the visible region. Although autologous skin grafting remains the gold standard of management, bioengineered skin substitutes such as cultured autologous and allogeneic keratinocyte grafts, composites, cellular and acellular matrices, and living skin substitutes, also have been shown to stimulate healing by acting as an occlusive dressing while releasing tissue growth factors and cytokines.

Elective, regional lymph node dissection has been suggested because of the proportionally elevated rate of lymph node metastasis, especially when lymph nodes are palpable or demonstrated with imaging techniques (as in our case), while it remains a controversial procedure in the absence of palpable nodes [12].

Finally, limb amputation may be recommended for recurrent disease, or when a Marjolin's ulcer and related disorders are associated with underlying osteomyelitis, when wide local excision will cause or will not outweigh a major functional disability. From an oncological standpoint, amputation does not seem to be superior to wide local excision, in terms of global disease outcome [20].

A long-term follow-up is recommended in all cases of Marjolin's disease and associated diseases leading to the development of an aggressive SCC. The majority of available patient series indicate that the incidence of relapse ranges from $20 \%$ to $50 \%$, and a 1980 study recorded that $98 \%$ of all recurrences were observed within three years of excision [21]. Most disease recurrences are regional in site, but metastases to the brain, liver, lung, kidney, and distant lymph nodes have been also reported. Novick recorded a $54 \%$ rate of metastases 
from lower limbs, and this rate was more than twice the metastatic index from any other primary site of "secondary" SCC [22]. In the same study, the overall threeyear survival rate of involved patients was quite low (66\%) [22]. Barr and Menard [20] reported a comparable five-year survival rate of $60 \%$ after wide excision, rising to $69 \%$ after limb amputation. If regional lymph nodes are interested (as happened in our patient), the three-year survival rate is expected to drop to around one half (approximately $35 \%$ ) [20]. Unfortunately, no sufficient evidence is present in the literature regarding the role of radiotherapy and chemotherapy in the management of this disease, so that the follow-up of our patient after his surgical intervention remains unpredictable.

\section{CONCLUSION}

In conclusion, the presence of chronic ulcers and draining sinuses after orthopedic surgery may prompt a rare case of Marjolin's ulcer or related disorders, including progression towards an aggressive SCC. Awareness about this disease and its complications and outcome, and an elevated suspicion risk are essential, when chronic cutaneous ulcers are present and infectious or bleedy drainages, increased size, or pain persist in a mid- longterm time span. A prompt diagnosis and management of these slow-progressing, but ultimately potentially life-threatening, malignancies remains a mandatory behavior for all interested specialists and health caregivers working with patients at risk for malignant transformation of chronic cutaneous lesions.

Key Points

- Patients affected by chronic osteomyelitis should be considered at risk for local and disseminated cancerogenesis

- The presence of chronic fistulae increases the risk of this complication

- These patients should be treated by a multidisciplinary approach

- Repeated skin biopsies and histopathology are mandatory

\section{Funding}

This article has been published without the support of sponsors.

Conflicts of interests

The authors declare they have no competing financial interests concerning the topics of this article.

\section{REFERENCES}

1. Wallingford SC, Olsen CM, Plasmeijer E, et al. Skin cancer arising in scars: a systematic review. Dermatol Surg 2011; 37: 1239-44; https://doi.org/10.1111/j.1524-4725.2011.02060.x

2. Mercuri SR, Brianti P, Rizzo N, et al. Basal cell carcinoma in post-traumatic scar successfully treated with thulium laser and photodynamic therapy. G Ital Dermatol Venereol 2018; (Mar 6) (Epub ahead of print)

3. Esther RJ, Lamps L, Schwartz HS. Marjolin's ulcers: secondary carcinomas in chronic wounds. J South Orthop Assoc 1999; 8: 181-7

4. Phillips TJ, Salman SM, Chawan J, et al. Burn scar carcinoma. Diagnosis and management. Dermatol Surg 1998; 24: 561-5; https://doi.org/10.1016/S1076-0512(98)00022-3; https://doi. org/10.1111/j.1524-4725.1998.tb04207.x

5. Simmons MA, Edwards JM, Nigam A. Marjolin's ulcer presenting in the neck.J Laryngol Otol 2000; 114: 980-2; https://doi.org/10.1258/0022215001904545

6. Steffen C. The man behind the eponym. Jean-Nicolas Marjolin. Am J Dermatopathol 1984; 6: 163-5; https://doi.org/10.1097/00000372-198404000-00009

7. Chong AJ, Klein MB. Images in clinical medicine. Marjolin's ulcer. N Engl J Med 2005; 352: e9; https://doi.org/10.1056/NEJMicm040020

8. Bartle EJ, Sum JH, Wanh XW, et al. Cancers arising from burn areas. A literature review of twenty-one cases. J Burn Care Rehabil 1990; 11: 46-9; https://doi.org/10.1097/00004630199001000-00010 
9. Lifeso RM,Bull CA. Squamous cell carcinoma of the extremities. Cancer 1985; 55: 2862-7; https:// doi.org/10.1002/1097-0142(19850615)55:12<2862::AID-CNCR2820551226>3.0.CO;2-H

10. Fleming MD, Hunt JL, Purdue GF, et al. Marjolin's ulcer: a review and re-evaluation of a difficult problem. J Burn Care Rehabil 1990; 11: 460-9; https://doi.org/10.1097/00004630199009000-00016

11. Smidt LS, Smidt LF, Chedid MB, et al. Radical surgical treatment for Marjolin ulcer occurring after chronic osteomyelitis. South Med J 2005; 98: 1053; https://doi.org/10.1097/01. smj.0000182509.78816.7b

12. Giunti A, Laus M.Tumori maligni in osteomielite cronica. Presentazione di 39 casi, 26 controllati a distanza. Giornale Italiano di Ortopedia e Traumatologia 1978; 4: 167-77

13. Campanacci M. Carcinomas and sarcomas on chronic osteomyelitis. In: Campanacci M (Ed.). Bone and soft tissues tumors. Bologna: Springer-Verlag - Gaggi, 1990, 661-664; https://doi. org/10.1007/978-3-662-29279-2_45

14. Manfredi R, Sabbatani S, Nanetti A, et al. A puzzling microbiological and clinical discrepancy in the management of acute, severe skin-soft tissue and joint staphylococcal infection. In vitro antimicrobial susceptibility to glycopeptides, versus in vivo clinical efficacy of linezolid alone. Infez Med 2006; 14: 157-64

15. Bostwick,J 3rd, Pendrgrast WJ Jr, Vasconez LO. Marjolin's ulcer: an immunologically privileged tumor? Plast Reconstr Surg 1976; 57: 66-9; https://doi.org/10.1097/00006534-197601000-00013

16. Cruickshank AH, McConnell EM, Miller DG. Malignancy in scars, chronic ulcers and sinuses. J Clin Pathol 1963; 16: 573-80; https://doi.org/10.1136/jcp.16.6.573

17. Trabucchi E, Pace M, Gabrielli L, et al. The trophic venous ulcer. The physiopathological, microbiological and pharmacological aspects. Minerva Cardioangiol 1994; 42: 43-50

18. Hill BB, Sloan DA, Lee EY, et al. Marjolin's ulcer of the foot caused by nonburn trauma. South Med J 1996; 89: 707-10; https://doi.org/10.1097/00007611-199607000-00011

19. Paredes F. Marjolin ulcer. Acta Med Port 1998; 11: 185-7

20. Barr LH, Menard JW. Marjolin's ulcer. The LSU experience. Cancer 1983; 52: 173-5; https:// doi.org/10.1002/1097-0142(19830701)52:1<173::AID-CNCR2820520131>3.0.CO;2-6

21. Ames FC, Jickey RC. Squamous cell carcinoma of the skin of the extremities. Int Adv Surg Oncol 1980; 3: 179-99

22. Novick M, Gard DA, Hardy SB, et al. Burn scar carcinoma: a review and analysis of 46 cases. JTrauma 1977; 17: 809-17; https://doi.org/10.1097/00005373-197710000-00010 